\title{
Two-Dimensional Numerical Simulation of Fermi- Level Pinning Phenomena Due to DX Centers in AlGaAs/GaAs HEMT's
}

HIROSHI MIZUTA, KEN YAMAGUCHI, MEMBER, IEEE, MASAO YAMANE, TOMONORI TANOUE, AND SUSUMU TAKAHASHI, MEMBER, IEEE

\begin{abstract}
Fermi-level pinning phenomena due to DX centers in AlGaAs /GaAs HEMT's are analyzed using two-dimensional numerical simulation based on a drift-diff usion model. A DX center model is introduced assuming Fermi-Dirac statistics for ionized donor density with the aluminum mole fraction dependence of the deep-donor energy level. The calculated results reveal that the decrease in transconductance of AlGaAs/GaAs HEMT's in a high gate-bias region is caused by the existence of DX centers. This is because the Fermi level is pinned at deep donor levels in the n-AIGaAs layer. Furthermore, the superiority of AIGaAs/InGaAs pseudomorphic HEMT's is discussed in terms of the Fermi-level pinning.
\end{abstract}

\section{INTRODUCTION}

$\mathrm{T}$ HE existence of DX centers [1], [2] in Si-doped $\mathrm{Al}_{x} \mathrm{Ga}_{1-x}$ As layers grown by $\mathrm{MBE}$ has been one of the most serious problems in developing heterostructured high-speed devices utilizing GaAs / AlGaAs systems. For example, persistent photoconductivity [1] in high electron mobility transistors (HEMT's) [3], [4], saturable pulsation of laser diodes [5], and persistent photocapacitance in $\mathrm{p}-\mathrm{n} \mathrm{GaAs} / \mathrm{AlGaAs}$ heterojunction diodes [6] are attributed to DX centers. In AlGaAs/GaAs HEMT's, two-dimensional electron gas (2DEG) formed at the Al$\mathrm{GaAs} / \mathrm{GaAs}$ heterointerface is used as a channel. DX centers have a large influence on the electron transfer between AlGaAs and GaAs layers [7]-[9], resulting in the saturation in sheet carrier density of 2DEG [10]. Therefore, it is important to suppress the influence of DX centers in order to obtain further improvement in $\mathrm{Al}$ $\mathrm{GaAs} / \mathrm{GaAs}$ HEMT performance.

Up to now, two-dimensional device simulators based on a classical drift-diffusion model have been used as powerful design tools. For the past few years, two-dimensional numerical models for HEMT's [11]-[13] have been developed based on the drift-diffusion approach. In addition, the velocity overshoot effect [12] and the surface Fermi-level pinning effect [13] have been investigated in AlGaAs / GaAs HEMT's. Recently, several authors [14]-[16] have reported one-dimensional analysis of charge control in HEMT's, taking DX centers into ac-

Manuscript received December 6, 1988; revised March 20, 1989. The authors are with the Central Research Laboratory, Hitachi, Ltd., Kokubunji, Tokyo 185, Japan.

IEEE Log Number 8929683 count. Park et al. [15] have shown good agreement between the calculated and experimental gate capacitancevoltage characteristics of AlGaAs /GaAs HEMT's. However, these calculations have been performed under a zero source-drain bias condition, and two-dimensional distributions of electrostatic potential and electron current in submicrometer-gate HEMT's have not been taken into consideration.

The present paper reports a full two-dimensional analysis of Fermi-level pinning phenomena due to DX centers in AlGaAs/GaAs HEMT's. We introduce a DX center model where the energy of deep donor levels varies with aluminum mole fraction. DX centers are shown to pin the Fermi level, thus greatly decreasing the transconductance of AlGaAs/GaAs HEMT's. Furthermore, the superior performance of InGaAs/AlGaAs pseudomorphic HEMT's as compared to that of conventional AlGaAs / GaAs HEMT's is discussed in terms of the disappearance of the Fermi-level pinning phenomena.

\section{Device Modeling}

The motion of carriers in HEMT's is analyzed by a classical drift-diffusion approach. The dependent variables are the electron density $n$, the hole density $p$, and the electrostatic potential $\psi$. Basic equations are described as follows:

1) Poisson equation

$$
\nabla \cdot(\epsilon \nabla \psi)=-q\left(N_{D}^{+}-N_{A}^{-}+p-n\right)
$$

where $q$ is the magnitude of an electron charge, $\epsilon$ is the dielectric constant, and $N_{D}^{+}$and $N_{A}^{-}$are the concentrations of ionized donors and acceptors. It is assumed that donors and acceptors in GaAs layers are fully ionized, except that the ionized donor density in n-AlGaAs layers depends on the aluminum mole fraction as explained later.

2) Current continuity equation

$$
\begin{aligned}
& \nabla \cdot J_{n}=q \cdot R \\
& \nabla \cdot J_{p}=-q \cdot R
\end{aligned}
$$

where $J_{n}$ and $J_{p}$ are the electron current density vector and the hole current density vector, and $R$ is the ShockleyRead-Hall recombination rate with an assumed carrier lifetime of $1.0 \times 10^{-9} \mathrm{~s}$. 


\section{3) Current density equation}

$$
\begin{aligned}
& J_{n}=-q \mu_{n} n \nabla \phi_{n} \\
& J_{p}=-q \mu_{p} p \nabla \phi_{p}
\end{aligned}
$$

where $\mu_{n}$ and $\mu_{p}$ are the carrier mobilities for electrons and holes, and $\phi_{n}$ and $\phi_{p}$ are the electron and hole quasi-Fermi potentials, respectively. These equations are discretized by the finite-difference method and solved self-consistently using the Gummel scheme [17].

The electron and hole densities are given by the following expressions:

$$
\begin{aligned}
& n=n_{i} \cdot \exp \left[\left\{\left(\psi+\theta-\gamma_{n}\right)-\phi_{n}\right\} / V_{T}\right] \\
& p=n_{i} \cdot \exp \left[\left\{\phi_{p}-\left(\psi+\theta-\gamma_{p}\right)\right\} / V_{T}\right]
\end{aligned}
$$

where $n_{i}$ is the intrinsic carrier density, $V_{T}$ is the thermal voltage $k_{B} T / q$, and $\gamma_{n}$ and $\gamma_{p}$ are degeneracy parameters to take Fermi-Dirac statistics into account [17]. The band parameter $\theta$ is defined as the energy difference between the vacuum level and the intrinsic Fermi level

$$
\theta=-\psi_{0}+\chi+\frac{E_{g}}{2}-\frac{V_{T}}{2} \cdot \ln \frac{N_{v}}{N_{c}}
$$

where $\psi_{0}$ is the potential reference, $\chi$ is the electron affinity, $E_{g}$ is the bandgap, and $N_{c}$ and $N_{v}$ are the effective densities of states in the conduction band and valence band, respectively.

The DX center model is introduced assuming FermiDirac statistics for an ionized deep donor density in $\mathrm{n}-\mathrm{Al}_{x} \mathrm{Ga}_{1-x}$ As layers. The ionized donor density in $\mathrm{n}$-AlGaAs layers is given by

$$
\begin{aligned}
& N_{D}^{+}(x) \\
& =N_{D} \frac{1}{1+g \cdot \exp \left[-\left(E c-\Delta E_{D}(x)-\phi_{n}\right) / k_{B} T\right]}
\end{aligned}
$$

where the degeneracy factor of the Si donor levels $g$, which equals 2 , and the energy $\Delta E_{D}$ are dependent on the Al mole fraction $x$. The functional formula $\Delta E_{D}(x)$ is adjusted to fit the experimental data by Ishikawa et al. [2]. For example, $\Delta E_{D}$ measured from the conduction band edge is assumed to be $60 \mathrm{meV}$ at $x=0.3$. To contrast the Fermi-level pinning phenomena caused by DX centers with Fermi-level behaviors in non-DX-center structures, a perfect ionization model is introduced as a reference; 100 percent ionization of donors in n-AlGaAs layers is assumed as for those in $\mathrm{n}$-GaAs layers.

$$
N_{D}^{+}(x)=N_{D} \text {. }
$$

Hereafter, these two models (i.e., (9) and (10)) are used to analyze the Fermi-level pinning due to DX centers in HEMT's.

The Al mole fraction dependence and the doping concentration dependence of carrier mobilities are considered according to the experimental data of Neumann and Flohrer [18]. The conduction band and valence band discon- tinuities $\Delta E_{c}$ and $\Delta E_{v}$, respectively, in the GaAs / AlGaAs heterostructure are assumed to be 60 and 40 percent of the $\Gamma$-band energy gap difference, respectively.

The electron velocity overshoot effect is approximately introduced using the following piecewise linear velocityfield relation [12]:

$$
\begin{aligned}
v_{n}(E)= & \mu_{n} \cdot E, \quad\left(E \leqq v_{s} / \mu_{n}\right) \\
& v_{s}, \quad\left(E>v_{s} / \mu_{n}\right)
\end{aligned}
$$

where the saturation velocity $v_{s}$ is estimated through quantitative comparison with experimental data as discussed later.

\section{Calculated Results}

\section{A. Influence of DX Centers on AlGaAs / GaAs HEMT Performance}

Fig. 1 shows a schematic cross section of the analyzed $0.5-\mu \mathrm{m}$-gate HEMT. It has a $0.015-\mu \mathrm{m}$-thick undoped AlGaAs surface layer [19], a $0.025-\mu \mathrm{m}$-thick highly doped n-AlGaAs layer, and a $0.002-\mu$ m-thick undoped AlGaAs buffer layer. The doping concentration in each layer used for the calculation is listed in Table I. The source and drain alloy regions are modeled by heavily doped $\mathrm{n}^{+}$-GaAs, and the semi-insulating substrate by a lightly doped $\mathrm{p}^{-}$-GaAs layer. The electron mobility in the slightly doped $\mathrm{p}^{-}$-GaAs channel is assumed to be 7200 $\mathrm{cm}^{2} /(\mathrm{V} \cdot \mathrm{s})$, and that in the n-AlGaAs layer 1500 $\mathrm{cm}^{2} /(\mathrm{V} \cdot \mathrm{s})$ according to the mobility model described in Section II. The Schottky-barrier height of the gate electrode is assumed to be $0.8 \mathrm{~V}$.

Fig. 2 shows the two-dimensional electrostatic potential distribution and electron current distribution near the un-AlGaAs $/ \mathrm{p}^{-}$-GaAs heterojunction calculated using the DX center model (see (9)). The bias conditions are source-drain voltage $V_{d s}=1.0 \mathrm{~V}$ and source-gate voltage $V_{g s}=0 \mathrm{~V}$. The length of each arrow shown in the figure is in proportion to the magnitude of the current density at that point. The electron current flows along the un-Al$\mathrm{GaAs} / \mathrm{p}^{-}$-GaAs heterointerface where the two-dimensional electron gas is formed. It should be noted that the current component due to low-mobility electrons in the n-AlGaAs layer, i.e., so-called parallel conduction, is negligible. The thickness and doping concentration of this layer are designed so that a charge-neutral region does not exist in this region.

The full transistor characteristics of the HEMT with a gate width of $200 \mu \mathrm{m}$ calculated at $300 \mathrm{~K}$ are shown in Fig. 3; (a) shows results using the DX center model, and (b) using the perfect ionization model. In these calculations, the saturation velocity of electrons $v_{s}$ is assumed to be $2.0 \times 10^{7} \mathrm{~cm} / \mathrm{s}$. The validity of this estimation is checked in the next section. It is found that the saturation current calculated using the DX center model is much smaller than that using the perfect ionization model.

In Figs. 4(a) and (b), drain current and transconductance calculated at drain bias $V_{d s}=1.2 \mathrm{~V}$ are shown as functions of gate bias. Calculated results using the DX 


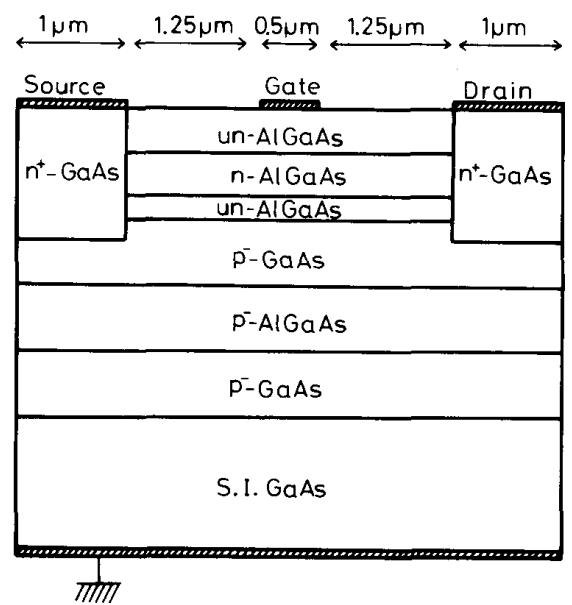

Fig. 1. A schematic cross-sectional view of the analyzed $0.5-\mu \mathrm{m}$-gate AlGaAs/GaAs HEMT.

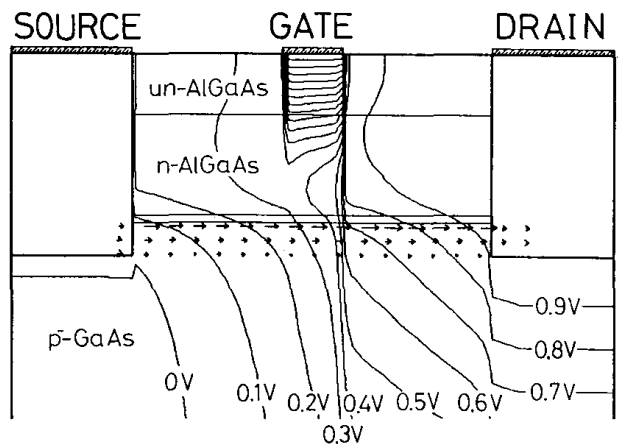

Fig. 2. Two-dimensional electrostatic potential distribution and electron current distribution near the AlGaAs / GaAs heterojunction for $V_{d s}=1.0$ $\mathrm{V}$ and $V_{g s}=0 \mathrm{~V}$. Electrostatic potentials are shown by contour lines with an interval of $0.1 \mathrm{~V}$.

TABLE I

Doping Concentration in Each Layer Used for Calculations

\begin{tabular}{c|c}
\hline Layer & Doping concentration $\left(\mathrm{c} \mathrm{m}^{-3}\right)$ \\
\hline un $\left(\mathrm{p}^{--}\right)-\mathrm{AlGaAs}$ & $1 \times 10^{13}$ \\
\hline $\mathrm{n}^{+}-\mathrm{AlGaAs}$ & $2 \times 10^{1 \mathrm{~s}}$ \\
\hline un $\left(\mathrm{p}^{--}\right)-\mathrm{AlGaAs}$ & $1 \times 10^{13}$ \\
\hline $\mathrm{p}^{-}-\mathrm{GaAs}$ & $1 \times 10^{14}$ \\
\hline $\mathrm{p}^{-}-\mathrm{AlGaAs}$ & $1 \times 10^{14}$ \\
\hline $\mathrm{p}^{-}-\mathrm{GaAs}$ & $1 \times 10^{14}$ \\
\hline S. I. GaAs & $1 \times 10^{\circ}$ \\
\hline Source, Drain n+GaAs & $2 \times 10^{10}$ \\
\hline
\end{tabular}

center model (solid lines) are compared to those using the perfect ionization model (dashed lines). As shown in Fig. 4(a), the drain currents calculated using these two models are nearly equal at the threshold voltage, but the difference between them increases when the gate bias approaches zero. The drain current using the DX center

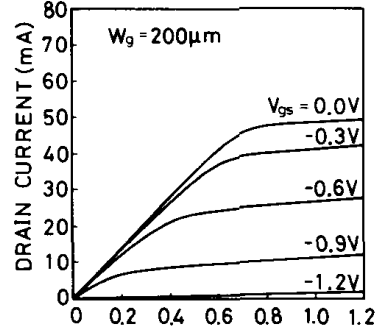

(a)
SOURCE-DRAIN VOLTAGE $(V)$

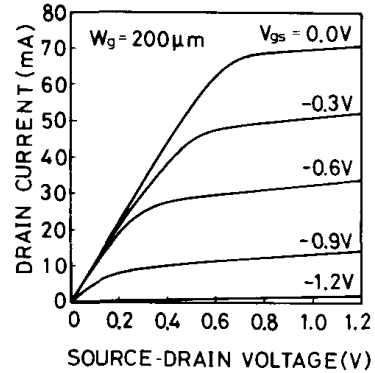

(b)
Fig. 3. The full transistor characteristics of the HEMT with a gate width of $200 \mu \mathrm{m}$ calculated at $300 \mathrm{~K}$. (a) Using the DX center model. (b) Using the perfect ionization model.

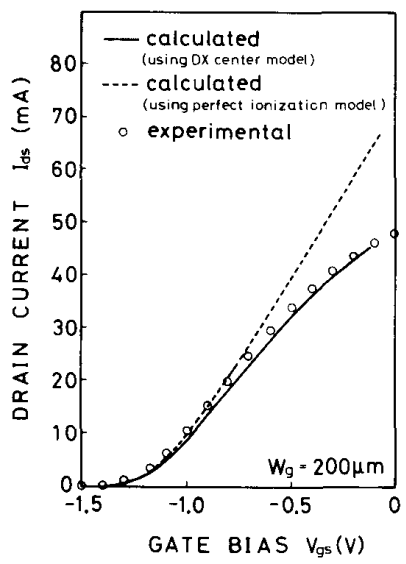

(a)

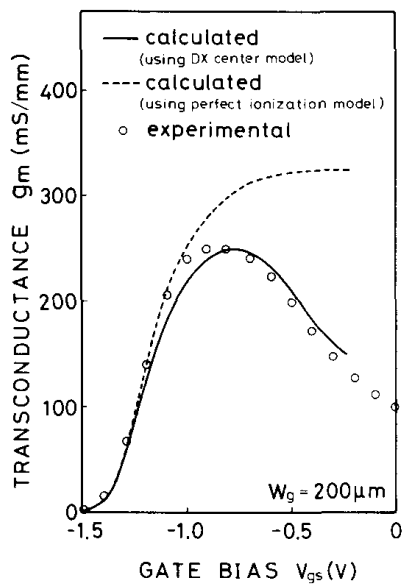

(b)

Fig. 4. Gate bias dependence of calculated and experimental (a) drain current $I_{d s}$ and (b) transconductance $g_{m}$. Solid lines represent the calculated results using the DX center model, and broken lines using the perfect ionization model. The experimental data are shown by open circles.

model tends to saturate, and the transconductance falls off rapidly near $V_{g s}=0 \mathrm{~V}$ as shown in Fig. 4(b). These results show excellent agreement, quantitatively and quali- 
tatively, with the experimental data (open circles), which are obtained from fabricated $0.5-\mu \mathrm{m}$-gate HEMT's with the same structural parameters as those used in the calculations.

As shown above, DX centers in the n-AlGaAs layer largely decrease the transconductance of $\mathrm{AlGaAs} / \mathrm{GaAs}$ HEMT's. Physically speaking, such a decrease in transconductance can be explained by Fermi-level pinning due to DX centers. This phenomenon is clarified in Section IV-A. Furthermore, the superior features of AlGaAs/InGaAs pseudomorphic HEMT's are discussed from the viewpoint of Fermi-level pinning in Section IVB.

\section{B. Velocity Overshoot Effect in AlGaAs/GaAs HEMT's}

For the two-dimensional electron gas at the AlGaAs / GaAs heterointerface, several Monte Carlo simulations have been carried out to obtain the electron velocity-electric field relation. For example, a calculated drift velocity of $1.5 \times 10^{7} \mathrm{~cm} / \mathrm{s}$ at an electric field of $5 \mathrm{kV} / \mathrm{cm}$ has been reported for a uniform two-dimensional electron gas at $300 \mathrm{~K} \mathrm{[20].} \mathrm{In} \mathrm{the} \mathrm{present} \mathrm{calculation,} \mathrm{the} \mathrm{piece-}$ wise velocity-field relation is employed. In this section, the saturation velocity $v_{s}$ is estimated from the calculated drain current and transconductance values.

Fig. 5(a) shows the calculated drain current as a function of gate bias with various values of $v_{s}$ (solid lines) and experimental values (open circles). In the same way, Fig. 5(b) shows the calculated transconductances and the experimental values. From these figures, it is inferred that $v_{s}=2.0 \times 10^{7} \mathrm{~cm} / \mathrm{s}$, which is consistent with the values reported so far [16], [19], [21], [22]. This value seems to be slightly larger than that estimated from the Monte Carlo simulations. In the HEMT structure, the electrostatic potential steeply drops near the drain side edge of the gate electrode, as shown in Fig. 2. A high electric field appears over a very short length (about $0.05 \mu \mathrm{m}$ ) of the channel, and the maximum electric field in this region is higher than $20 \mathrm{kV} / \mathrm{cm}$. The electron velocity in this small region is inferred to exceed the drift velocity in a uniform channel obtained from Monte Carlo simulations. The saturation velocity in (11) can be interpreted as an average over the whole channel. Considering the local velocity overshoot in the channel, the $v_{s}$ value of $2.0 \times 10^{7} \mathrm{~cm} / \mathrm{s}$ estimated in this section is considered to be adequate.

\section{Discussion}

\section{A. Fermi-Level Pinning Due to DX Centers}

In this section, Fermi-level pinning due to DX centers is discussed. The calculated sheet electron concentration of the two-dimensional electron gas $n_{s}^{2 \mathrm{DEG}}$ is shown in Fig. 6 as a function of gate bias. The increase in $n_{s}^{2 D E G}$ calculated using the DX center model (solid line) is suppressed near zero bias, i.e., the change in $n_{s}^{2 \mathrm{DEG}}$ by gate bias becomes'small. This suppression of maximum $n_{s}^{2 \mathrm{DEG}}$ brings about the decrease in transconductance described in Section III-A. In the perfect ionization model (broken line),

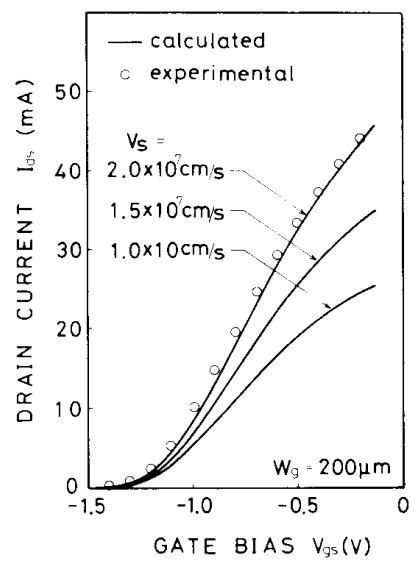

(a)

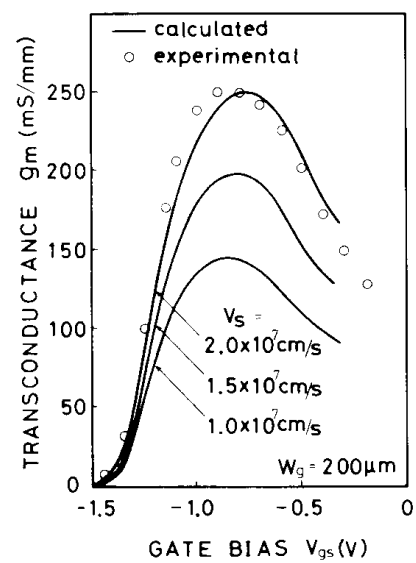

(b)

Fig. 5. Calculated (a) drain current and (b) transconductance as functions of gate bias with various values of $v_{s}$. Experimental results are plotted by open circles.

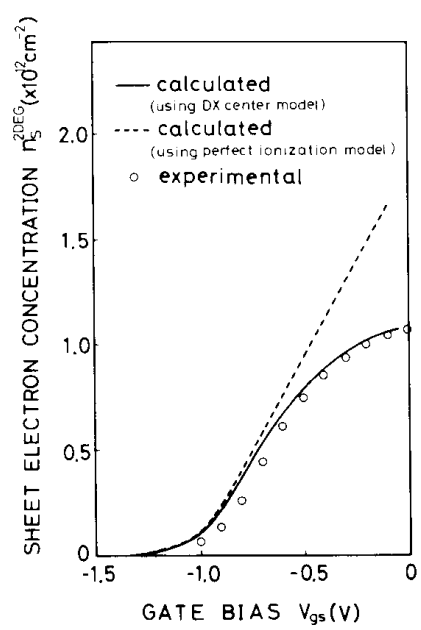

Fig. 6. Calculated electron concentration $n_{s}^{2 D E G}$ accumulating at the heterointerface as a function of gate bias. Solid lines represent the calculated results using the DX center model, and broken lines using the perfect ionization model. Experimental results are plotted by open circles. 
$n_{s}^{2 \mathrm{DEG}}$ depends almost linearly on gate bias, and the maximum $n_{s}^{2 \mathrm{DEG}}$ is about twice that using the DX center model. Experimental values are shown with open circles, and these agree well with the calculated values using the DX center model.

Conduction band-edge profiles as a function of depth are shown in Fig. 7. The calculated results using the DX center model and the perfect ionization model at thermal equilibrium are shown in Fig. 7(a) and (b), respectively. The deep donors in the n-AlGaAs layer, namely DX centers, are indicated by the broken line in Fig. 7(a). The Fermi level (thin solid line) shown in Fig. 7(a) is located near the DX centers in the n-AlGaAs layer. In contrast, that shown in Fig. 7(b) is located near the conduction band edge. It should be noted that DX centers act as effective conduction band edge.

The calculated electron quasi-Fermi potential $\phi_{n}$ measured from the conduction-band minimum is shown in Fig. 8 as a function of gate bias. For gate bias $V_{g s}<-1.0$ $\mathrm{V}$, the electron quasi-Fermi potential $\phi_{n}$ calculated using the DX center model (solid line) is almost the same as that calculated using the perfect ionization model (broken line). They begin to separate at about $V_{g s}=-0.8 \mathrm{~V}$. Such $\phi_{n}$ differences give rise to the difference between the $n_{s}^{2 \mathrm{DEG}}$ values obtained from the two model calculations (see Fig. 6). At near zero bias, $\phi_{n}$ calculated using the DX center model approaches the deep-donor energy level $\Delta E_{D}$ and not the conduction band edge. This is the Fermilevel pinning due to DX centers, which suppresses the maximum $n_{s}^{2 \mathrm{DEG}}$ and $g_{m}$.

Electron distributions are shown in Fig. 9 for the same bias conditions used in Fig. 7. It can be seen that the electron concentration of the 2DEG calculated using the DX center model (solid line) becomes smaller than that using the perfect ionization model (broken line) because of this Fermi-level pinning phenomenon.

As shown above, DX centers in the $\mathrm{n}$-AlGaAs layer cause Fermi-level pinning resulting in a large decrease in transconductance of AlGaAs/GaAs HEMT's. In Section IV-B, an AlGaAs/InGaAs pseudomorphic HEMT structure is analyzed in terms of the Fermi-level pinning phenomenon.

\section{B. Discussion on Pseudomorphic AlGaAs /InGaAs HEMT's}

Recently, much attention has been paid to AlGaAs/InGaAs pseudomorphic HEMT's [23], [24]. In this structure, a large conduction-band discontinuity can be obtained by using a small $\mathrm{Al}$ mole fraction $(x<0.2)$ where DX centers are not created in $\mathrm{n}-\mathrm{AlGaAs}$ layers. Thus, the AlGaAs/InGaAs pseudomorphic HEMT structure is attractive for eliminating device performance degradation caused by DX centers. In this section, we discuss the performance of AlGaAs/InGaAs pseudomorphic HEMT's compared with that of conventional AlGaAs / GaAs HEMT's.

The AlGaAs/InGaAs pseudomorphic HEMT analyzed here has the same structure as the AlGaAs/GaAs HEMT

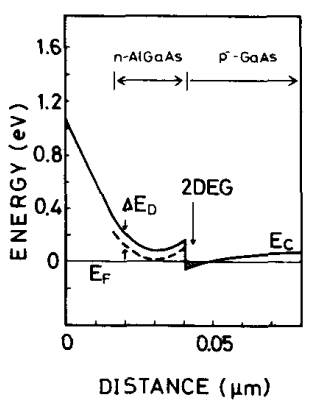

(a)

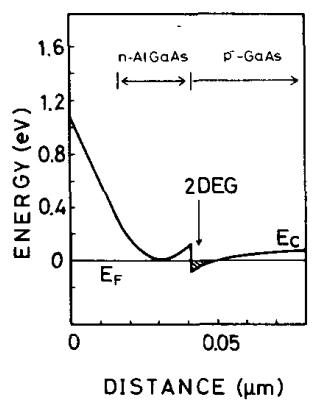

(b)
Fig. 7. Calculated energy band diagram as a function of depth in thermal equilibrium. (a) Using the DX center model. (b) Using the perfect ionization model.

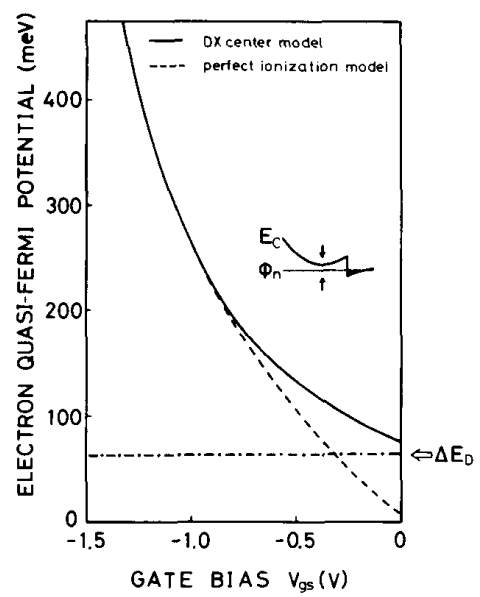

Fig. 8. Calculated electron quasi-Fermi potential measured from conduction-band minimum as a function of gate bias. Solid lines represent the calculated results using the DX center model, and broken lines using the perfect ionization model.

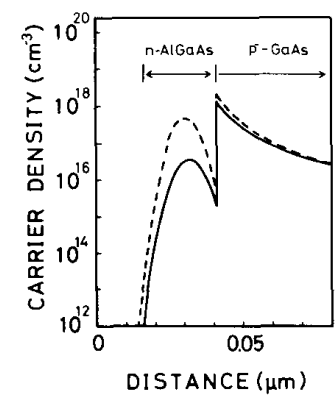

Fig. 9. Calculated electron distribution as a function of depth in thermal equilibrium.

investigated above except for the following two points. The $\mathrm{Al}$ mole fractions of the $\mathrm{Al}_{0.3} \mathrm{Ga}_{0.7}$ As layers are all changed with 0.15 , and $\mathrm{p}^{-}$-GaAs layer where the twodimensional electron gas is formed is replaced by a $\mathrm{p}^{-}$-InGaAs layer; the In mole fraction is determined to be 0.25 so that the pinchoff voltage of the analyzed pseudomorphic HEMT is nearly equal to that of the $\mathrm{Al}$ - 
GaAs/GaAs HEMT. The energy bandgap shifts in the InGaAs layer due to elastic strain produced by the lattice mismatch is introduced according to [25] and [26]; the conduction band discontinuity in the $\mathrm{Al}_{0.15} \mathrm{Ga}_{0.85} \mathrm{As} /$ $\mathrm{In}_{0.25} \mathrm{Ga}_{0.75} \mathrm{As}$ heterostructure is estimated to be $231 \mathrm{meV}$. In the $\mathrm{p}^{-}-\mathrm{In}_{0.25} \mathrm{Ga}_{0.75} \mathrm{As}$ layer, the electron effective mass is assumed to be $0.056 m_{0}$ and the electron mobility is $7200 \mathrm{~cm}^{2} /(\mathrm{V} \cdot \mathrm{s})$, the same as for the $\mathrm{p}^{-}$-GaAs layer. In this calculation, we assume (9) for the ionized donor density in the $\mathrm{n}^{+}-\mathrm{Al}_{0.15} \mathrm{Ga}_{0.85} \mathrm{As}$ layer. The energy level of the Si donor, $\Delta E_{D}$, in the $\mathrm{n}^{+}-\mathrm{Al}_{0.15} \mathrm{Ga}_{0.85}$ As layer is 4 $\mathrm{meV}$ [2]. For electron saturation velocity in InGaAs layers, some experimental values have been reported [23], [27]. The purpose of this analysis is to investigate the Fermi-level pinning phenomena. Therefore, the same saturation velocity of $2.0 \times 10^{7} \mathrm{~cm} / \mathrm{s}$ is assumed so that we can directly compare device performance with and without the DX centers' effect; this assumption is considered to be valid, according to [28].

Figs. 10(a) and (b) shows drain current and transconductance as functions of gate bias. The solid lines represent the calculated results for the AlGaAs/InGaAs pseudomorphic HEMT, and the broken lines for the AlGaAs / GaAs HEMT. It should be noted that improvements in the drain current and transconductance of the AlGaAs / InGaAs pseudomorphic HEMT can be seen near $V_{g s}=0 \mathrm{~V}$. The reason for these improvements is that the Fermi level is not pinned at such deep levels as DX centers in the $\mathrm{n}-\mathrm{Al}_{0.3} \mathrm{Ga}_{0.7} \mathrm{As}\left(\Delta E_{D}=60 \mathrm{meV}\right)$ and can rise to near the conduction band edge. Owing to the disappearance of Fermi-level pinning, the maximum transconductance can be enhanced to $325 \mathrm{mS} / \mathrm{mm}$. This is almost the same value as obtained using the perfect ionization model in Section III (see broken line in Fig. 4(b)).

As discussed above, the Fermi-level pinning phenomena disappear in AlGaAs/InGaAs pseudomorphic HEMT's. It should be noted that the transconductance is enhanced even though the same electron mobility and saturation velocity are assumed in the InGaAs layer. This improvement results only from the disappearance of the Fermi-level pinning phenomena due to DX centers.

\section{Conclusion}

Fermi-level pinning phenomena due to DX centers in AlGaAs/GaAs HEMT's have been theoretically analyzed using two-dimensional numerical calculations based on the drift-diffusion model. The DX center model has been introduced assuming Fermi-Dirac statistics for ionized donor density with the $\mathrm{Al}$ mole fraction dependence of donor levels in n-AlGaAs layers. The calculated results using the DX center model are in excellent agreement with the experimental data. It has been revealed that the Fermi level is pinned by DX centers and that maximum transconductance of AlGaAs / GaAs HEMT's is very much decreased. The superiority of AlGaAs/InGaAs pseudomorphic HEMT's has been demonstrated. It has been shown that Fermi-level pinning phenomena disappear in this structure. The maximum transconductance of $\mathrm{Al}$ -

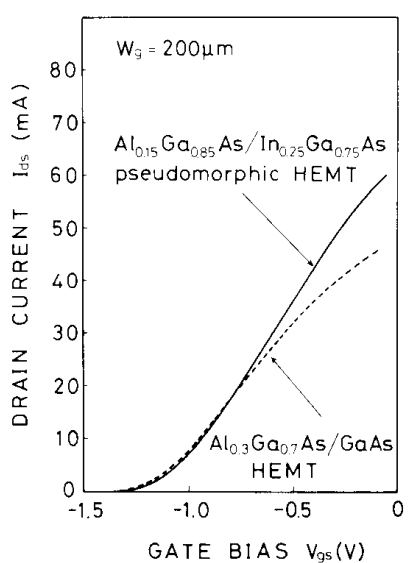

(a)

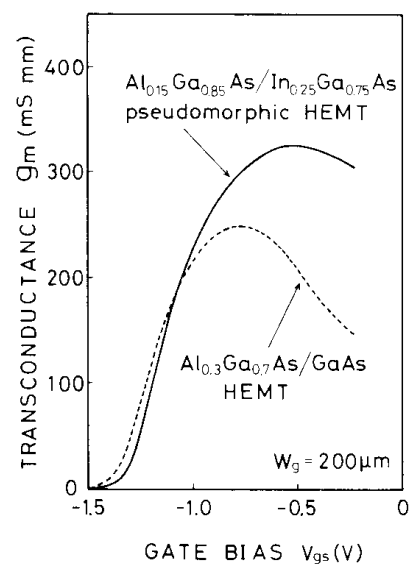

(b)

Fig. 10. Gate bias dependence of (a) drain current and (b) transconductance $g_{m}$. Solid lines represent the calculated results for the AlGaAs/InGaAs HEMT, and broken lines for the AlGaAs/GaAs HEMT.

GaAs / InGaAs pseudomorphic HEMT's is enhanced even though the same values of mobility and saturation velocity as used in the conventional AlGaAs/GaAs HEMT's are assumed.

\section{AKNOWLEDGEMENT}

The authors wish to thank Dr. T. Usagawa, Dr. Y. Uchida, and S. Goto for many helpful discussions, and Dr. H. Matsumura for his encouragement during the course of this work.

\section{REFERENCES}

[1] D. V. Lang, R. A. Logan, and M. Jaros, “Trapping characteristics and a donor-complex (DX) model for the persistent-photoconductivity trapping center in Te-doped $\mathrm{Al}_{x} \mathrm{Ga}_{1-x} \mathrm{As}$," Phys. Rev. B, vol. 19, pp. 1015-1030, 1979

[2] T. Ishikawa, J. Saito, S. Sasa, and S. Hiyamizu, "Electrical properties of Si-doped $\mathrm{Al}_{x} \mathrm{Ga}_{1-x}$ As layers grown by MBE,' Japan. $J$. Appl. Phys, vol. 21, no. 11, pp. L675-L676, 1982.

[3] T. Mimura, S. Hiyamizu, T. Fujii, and K. Nanbu, "A new field effect transistor with selectively doped $\mathrm{GaAs} / \mathbf{n}-\mathrm{Al}_{x} \mathrm{Ga}_{1-x}$ As heterojunctions,"' Japan. J. Appl. Phys., vol. 19, pp. L225-L227, 1980. 
[4] S. Hiyamizu, T. Mimura, T. Fujii, and K. Nanbu, "High mobility of two-dimensional electrons at the GaAs / n-AlGaAs heterojunction interface," Appl. Phys. Lett., vol. 37, pp. 805-807, 1980.

[5] J. L. Merz, J. P. van der Ziel, and R. A. Logan, "Saturable optical absorption of the deep Te-complex center in $\mathrm{Al}_{0.4} \mathrm{Ga}_{0.6} \mathrm{As}$," Phys. Rev. $B$, vol. 20 , pp. 654-663, 1979.

[6] M. I. Nathan, S. Tiwari, P. M. Mooney, and S. L. Wright, "DX centers in AlGaAs p-n heterojunctions and heterojunction bipolar transistors," J. Appl. Phys., vol. 62, pp. 3234-3236, 1987.

[7] P. Godts, E. Constant, J. Zimmermann, and D. Depreeuw, "Investigation of influence of DX centres on HEMT operation at room temperature,"' Electron. Lett., vol. 24, pp. 937-938, 1988.

[8] M. I. Nathan, P. M. Mooney, P. M. Solomon, and S. L. Wright, "Room-temperature electron trapping in $\mathrm{Al}_{0.35} \mathrm{Ga}_{0.65} \mathrm{As} / \mathrm{GaAs}$ modulation-doped field-effect transistors," Appl. Phys. Lett., vol. 47, pp. $628-630,1985$

[9] A. Kastalsky and R. A. Kiehl, " On the low-temperature degradation of (AlGa) As / GaAs modulation-doped field-effect transistors," IEEE Trans. Electron Devices, vol. ED-33, no. 3, pp. 414-423, 1986.

[10] K. Hirakawa, H. Sakaki, and J. Yoshino, "Concentration of electrons in selectively doped GaAlAs/GaAs heterojunction and its dependence on spacer-layer thickness and gate electric field," Appl. Phys. Lett., vol. 45, pp. 253-255, 1984.

[11] D. Loret, "Two-dimensional numerical model for the high electron mobility transistor," Solid-State Electron., vol. 30, no. 11, pp. 1197 1203, 1987.

[12] J. Yoshida and M. Kurata, "Analysis of high electron mobility transistors based on a two-dimensional numerical model," IEEE Electron Device Lett., vol. EDL-5, no. 12, pp. 508-510, 1984.

[13] J. Y.-F. Tang, "Two-dimensional simulation of MODFET and GaAs gate heterojunction FET's,' IEEE Trans. Electron Devices, vol. ED-32, no. 9, pp. 1817-1823, 1985.

[14] M. J. Moloney, F. Ponse, and H. Morkoc, "Gate capacitance-voltage characteristics of MODFET's: Its effect on transconductance," IEEE Trans. Electron Devices, vol. ED-32, no. 9, pp. 1675-1684, 1985.

[15] K. Park, H. B. Kim, and K. D. Kwack, "A model for the capacitance-voltage characteristics of MODFET's," IEEE Trans. Electron Devices, vol. ED-34, no. 12, pp. 2422-2427, 1987.

[16] Y. Ando and T. Itoh, "Analysis of charge control in pseudomorphic two-dimensional electron gas field-effect transistors," IEEE Trans. Electron Devices, vol. 35, no. 12, pp. 2295-2301, 1988.

[17] T. Ohtoshi et al., "A two-dimensional device simulator of semiconductor lasers," Solid-State Electron., vol. 30, no. 6, pp. 627-638, 1987.

[18] H. Neumann and U. Flohrer, "Electron mobility in $\mathrm{Al}_{x} \mathrm{Ga}_{1-x} \mathrm{As}$," Phys. Status Solidi, vol. 25a, pp. K145-K147, 1974.

[19] H. Hida, K. Ohata, Y. Suzuki, and H. Toyoshima, "A new low-noise AIGaAs/GaAs 2DEG FET with a surface undoped layer," IEEE Trans. Electron Devices, vol. ED-33, no. 5, pp. 601-607, 1986.

[20] K. Yokoyama and K. Hess, "Calculation of warm electron transport in AlGaAs/GaAs single heterostructures using a Monte Carlo method," J. Appl. Phys., vol. 59, no. 11, pp. 3798-3802, 1986.

[21] Y. Takanashi and N. Kobayashi, "AlGaAs/GaAs 2-DEGFET's fabricated from MO-CVD wafers," IEEE Electron Device Lett., vol. EDL-6, no. 3, pp. 154-156, 1985.

[22] W. P. Hong and P. K. Bhattacharya, "High-field transport in InGaAs/InAlAs modulation-doped heterostructures,'" IEEE Trans. Electron Devices, vol. ED-34, no. 7, pp. 1491-1495, 1987.

[23] A. Ketterson et al., "High transconductance InGaAs / AlGaAs pseudomorphic modulation-doped field-effect transistors," IEEE Electron Device Lett., vol. EDL-6, no. 12, pp. 628-630, 1985.

[24] T. Henderson et al., "Microwave performance of a quarter-micrometer gate low-noise pseudomorphic InGaAs/AlGaAs modulationdoped field effect transistor," IEEE Electron Device Lett., vol. EDL-7, no. 12 , pp. 649-651, 1986.

[25] H. Asai and K. Oe, "Energy band-gap shift with elastic strain in $\mathrm{Ga}_{x}$ In $_{1-x}$ P epitaxial layers on (001) GaAs substrates," $J$. Appl. Phys., vol. 54, no. 4, pp. 2052-2056, 1983.

[26] K. Nishi, K. Hirose, and T. Mizutani, "'Optical characterization of InGaAs-InAlAs strained-layer superlattices grown by molecular beam epitaxy," Appl. Phys. Lett., vol. 49, pp. 794-795, 1986.

[27] T. S. Henderson, W. T. Masselink, W. Kopp, and H. Morkoç, "Determination of carrier saturation velocity in high-performance $\mathrm{In}_{y} \mathrm{Ga}_{1-y} \mathrm{As} / \mathrm{Al}_{x} \mathrm{Ga}_{1-x} \mathrm{As}$ modulation-doped field-effect transistors ( 0 $\leqq y \leqq 0.2$ )," IEEE Electron Device Lett., vol. EDL-7, no. 5, pp. $228-290,1986$.
[28] L. W. Nguyen, D. C. Radulescu, P. W. Tasker, W. J. Schaff, and L. F. Eastman, "0.2- $\mu \mathrm{m}$ gate-length atomic-planar doped pseudomorphic $\mathrm{Al}_{0.3} \mathrm{Ga}_{0.7} \mathrm{As} / \mathrm{In}_{0.25} \mathrm{Ga}_{0.75}$ As MODFET's with $f_{T}$ over 120 GHz,', IEEE Electron Device Lett., vol. 9, no. 8, pp. 374-376, 1988.

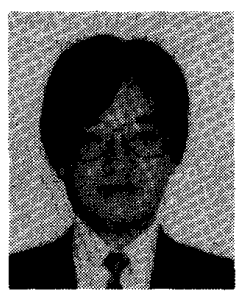

Hiroshi Mizuta was born in Kochi, Japan, in 1961. He received the B.S. and M.S. degrees in physics from Osaka University, Osaka, Japan, in 1983 and 1985 , respectively.

He joined the Central Research Laboratory, Hitachi Ltd., Tokyo, Japan, in 1985, and has been engaged in research on device physics, device modeling, simulation technology, and new functional devices. His current research interests are in theoretical analysis of quantum transport in multi-barrier heterostructures and two-dimensional numerical simulation of heterojunction devices affected by deep levels such as DX centers, EL2 donors, and interface trap levels.

Mr. Mizuta is a member of the Physical Society of Japan and the Japan Society of Applied Physics.

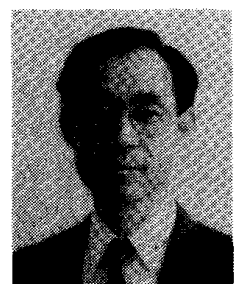

Ken Yamaguchi (M'76) was born in Tokyo, Ja pan, in 1947. He received the B.Sc. and M.Sc. degrees from Yokohama National University, Yokohama, Japan, in 1970 and 1972 , respectively, and the Ph.D. degree from the University of Tokyo, Tokyo, Japan, in 1980, all in electrical engineering.

He joined the Central Research Laboratory, Hitachi, Ltd., in 1972, and has been working in the field of semiconductor device design technologies. His current research interests are in device physics, device modeling, simulation technologies, and CAD systems for designing semiconductor devices and circuits.

Dr. Yamaguchi is a member of the Physical Society of Japan, the Japan Society of Applied Physics, the Institute of Electronics, Information and Communication Engineers of Japan, and the Electron Devices Society of the IEEE.

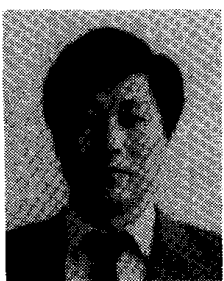

Masao Yamane was born in Okayama, Japan, in 1955. He received the B.Sc. degree in physics from Hiroshima University, Hiroshima, Japan, in 1979 and the master's and doctor's degrees in engineering from Osaka University, Osaka, Japan, in 1981 and 1984 , respectively.

He joined the Central Research Laboratory, Hitachi, Ltd., Tokyo, Japan, in 1984, and has been engaged in research on the design and fabrication of HBT's, low-noise 2DEGFET's, and low-noise MMIC's. His current research interests are the modeling of 2DEGFET's and designing ultra-small-size low-noise MMIC's using 2DEGFET's.

Dr. Yamane is a member of the Physical Society of Japan, the Japan Society of Applied Physics, and the Institute of Electronics, Information and Communication Engineers of Japan. 


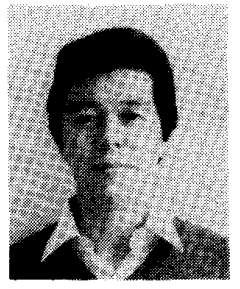

Tomonori Tanoue was born in Tokyo, Japan, in 1957. He received the bachelor's, master's, and doctor's degrees in engineering from the University of Tokyo, Tokyo, Japan, in 1980, 1982, and 1986, respectively.

Since he joined the Central Research Laboratory, Hitachi, Ltd., Japan, in 1986, his main concern has been the growth and fabrication of heterojunction devices such as MQW lasers, HBT's, heterojunction FET's, and resonant tunneling devices. He is currently working on new functional devices using resonant tunneling barriers.

Dr. Tanoue is a member of the Japan Society of Applied Physics and the Institute of Electronics, Information and Communication Engineers of Japan.

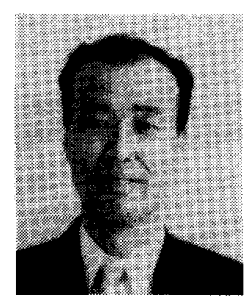

Susumu Takahashi (M'82) was born in Iwat:, Japan, in 1944. He received the B.Sc. degree : physics from Hirosaki University, Hirosaki, $\mathrm{J}_{\mathrm{i}}$ pan, in 1967 and the Ph.D. degree in electric il engineering from the University of Tokyo, Tokyo, Japan, in 1979.

Since 1967, he has been working for the Cel1tral Research Laboratory, Hitachi, Ltd., Tokys, Japan, on low-noise GaAs MESFET's, silicon dizvices, and GaAs LSI's. His current research interests are compound heterodevices and technology.

Dr. Takahashi is a member of the Japan Society of Applied Physics, th e Institute of Electronics, Information and Communication Engineers of $\mathrm{J}_{\mathrm{i}}$ pan, and the Electron Devices Society of the IEEE. 